\title{
A Review on Noise Reduction of Echo Cardiographic Images based on Temporal Information
}

\author{
Archana Sharma \\ M. Tech Scholar \\ NRI Institute of Information Science \& Technology \\ Bhopal, India
}

\author{
Swapnil Jain \\ H.O.D \\ NRI Institute of Information Science \& Technology \\ Bhopal, India
}

\begin{abstract}
Medical imaging is inserting a significant role in diagnosis the diseases and in image guided surgery. There are varied imaging modalities for various applications giving the anatomical and physiological conditions of the patient. Noise suppression of echocardiography images may be a difficult issue for correct and effective human interpretation and computer-assisted analysis. In spite of comprehensive speckle reduction ways, up to now there are few studies of de-noising echocardiography sequences supported temporal data. During this article, a quick and correct filter supported temporal data has been projected that permits the reduction of noise in echocardiography images. The projected methodology consists of smoothing intensity variation time curves (IVTC) assessed in every picture element. By filtering high-frequency elements of every temporal signal and so substitution the smooth signals in their positions, all pixels of all frames are often reconstructed during a parallel manner. The presentation of the projected methodology is evaluated and compared with seven alternative speckle-reduction filters. Judgment of the filters is predicated on a series of computer-simulated and real clinical images, and additionally on visual assessment by specialists. The experimental results show that the projected algorithmic rule is quick, less computationally demanding than alternative filters, and correct, additionally to preserving the edges of the images.
\end{abstract}

\section{Keywords}

MMSE (Minimizing the Mean-Square-Error)

\section{INTRODUCTION}

In a process of an ultrasound imaging system, if the envelope signal is captured before logarithmic compression, the speckle noise model could also be approximated as increasing noise. When logarithmic compression, the speckle noise becomes noise transforms into the classical signal within the additive noise model. Terribly near white Gaussian noise and also the multiplicative as declared by Parisa Gifani, heart and valve biodynamics and also the mechanisms making certain their correct functioning are controlled by several complicated multiscale factors including the surrounding hemodynamic setting. This paper addresses the matter of computing the blood motion flow field through the left heart chambers. We tend to believe that our computational approach is going to be useful for aiding the assessment of the heart dynamic and practical behavior. Such info are often exploited in surgical designing systems to assist predict the result of complicated reconstructive surgeries like cardiac valvuloplasty. This work may also be used to augment TEE rendering, and assist cardiologists and clinicians in higher assessing pathologies like heart valve regurgitation, by providing a better resolution and additional complete (i.e., full vectorial) image of the flow in comparison to the present Doppler echocardiography. TEE is that the imaging modality chosen for this work since it's versatile and non-ionizing and may be exploited each pre- or intra-operatively. Mathematical modeling of cardiac physiology and biodynamic was pioneered by Ritwik Kumar, World Health Organization introduced a way supported FluidStructure Interaction (FSI), termed the "immersed boundary" (IB) approach. in progress work is being pursued to increase IB and address a number of its reported limitations as well as the valve's infinitely thin structural approximation, with no slip, wherever the fluid is ideally viscous and can't admit discontinuities, and the shortcoming to model static loading, bending and shear behavior. as a result of the speckle texture usually contains info regarding the tissue texture, speckle isn't actually a noise. Unfortunately, however, the presence of speckle limits contrast resolution in diagnostic ultrasound imaging and additionally adversely affect the appliance of image process algorithms like edge detection, segmentation, tracking, and registration. Similarly, the variations caused by speckle are present in all cross-sectional views of echocardiography images. Speckle is distinguished and additional important than additive noise. Speckle should be suppressed to positively have an effect on subjective image quality and improve alternative image process applications. a substantial quantity of analysis makes an attempt to cut back or eliminate speckle. Current approaches fall under 2 classes. the primary class is based on using the phased data of RF signals; the second class includes post processing approaches. Most post-processing techniques are well developed and include native statistical filters, geometric filters, wavelet filters and anisotropic diffusion filters.

In the class of native statistical filters, a median filter is one among the simplest speckle reduction filters. During this filter, the central pixel of a moving window is replaced by the window's median. By presuming a multiplicative model of speckle noise, additional difficult statistical filters have developed, like the Lee filter and therefore the Kuan filter. These filters have similar structures, and therefore the Lee filter will be considered a specific version of the Kuan filter. each are moving-window-based filters and generate the output image by minimizing the mean-square-error (MMSE) criteria. Within the Lee filter, a linear approximation is formed for the multiplicative noise model. The difference between the Kuan and Lee filters is that within the Kuan filter the multiplicative noise is remodeled to signal-dependent additive noise and so a linear filter supported MMSE is performed. The Frost filter is an adaptive Wiener filter and relies on an assumption of stationary noise statistics and therefore the multiplicative noise model. This filter implements a weighted average of pixel values during a moving window within which the weights are negative exponential functions of the standard deviation of the noise.

\section{LITERATURE REVIEW}

Parisa Gifani et. al. [1] "Noise Reduction of Echo cardio 
graphic Images Based on Temporal Information" Noise repression of echocardiography descriptions is a demanding topic for perfect and efficient human explanation and computer-assisted investigation. In nastiness of complete speckle reduction methods, until at the moment there have been only some studies of de-noising echocardiography sequence based on sequential information. In this piece of writing, a fast and correct filter based on sequential information has been projected that enable the decrease of noise in echocardiography images. The projected method consists of smooth intensity variation time curves (IVTC) assess in all pixels. By filter high-frequency apparatus of each chronological indication and then replace the smooth signal in their position, all pixels of the entire frames can be reconstruct in a analogous manner. The presentation of the projected technique is evaluate and compare with seven extra specklereduction filter. Evaluation of the filters is base on a succession of computer-simulated and actual experimental descriptions, and also on visual estimation by expert. The experimental consequences show that the projected algorithm is fast, less computationally difficult than further filters, and precise, in addition to preserve the limits of the images. The purpose of this work is to de-noise echo cardio graphic images base on temporal information. In the pro-posed algorithm, the filter has been finished work on video frames which had been throughout reduction improvement and a nonlinear transform (close to the logarithmic transform) of the strength rate confirmation by a transducer. Accordingly, the noise model can be understood to be an additive noise model and the common LTI low-pass filter can contain the high-frequency noise components. In this paper, we implement a second-order low-pass Butterworth filter to decrease the noise in long-term temporal signals obtained from fixed coordinates of consecutive frames. In aspect, we qualified and choose the Butterworth filter from among other low-pass filters. The experienced filters were the second-order Bessel filter and hard thresholding of FFT coefficients. We obtained the best result from the Butterworth filter in terms of both quantitative metrics and computational expenditure.

C. B. Burckhardt et.al "Speckle in Ultrasound B-Mode Scans" [2] Ultrasound images obtained with a simple linear or sector scan show a granular appearance, called "speckle." This speckle is analyze. The cutbacks in speckle with the intention of know how to be obtained with a complex scan with greatest amplitude symbols is computed. The cutback in speckle is nearly as large as can be obtain with averaging. It depends on the quantity of self-determining amplitude standards that are measured. The requirement for the liberty of two amplitude value is derived, and thus a maximum value is given for the promising decrease in speckle. Speckle in ultrasound images has been analyzed for simple scans and for compound scans. It was especially shown that the improvement in SNR is almost as good for a "maximum amplitude writing" compound scan as for an "averaging": compound scan. In view of its other advantages the first mode of operation is to be preferred. It was then shown that the transducer has to be translated by about half its width when independent amplitude values are to be obtained. This places a definite limit on the possible improvement in SNR. The achievable enhancement is poorer in support of high-resolution (i.e., large aperture) transducers than for low-resolution (i.e., small aperture) transducers.

Khaled Z. Abd-Elmoniem, et. al. [3] "Real-Time Speckle Reduction and Coherence Enhancement in Ultrasound Imaging via Nonlinear Anisotropic Diffusion". The projected NCD representation combines three different models.
According to speckle amount and image anisotropy, the NCD representation changes with time from isotropic distribution through anisotropic coherent transmission to, finally, mean curvature motion. This arrangement maximally low-pass filter individuals parts of the image that keep up a correspondence to fully developed speckle, while substantially preserving information related with resolved-object structure. The projected execution algorithm utilizes a resourceful discretization method that allow for real-time execution on commercial system. The theory and execution of the new method are accessible and conventional by means of specter and clinical ultrasound images. In computation, the consequences as of earlier techniques are comparing with the new method to demonstrate its performance. A new nonlinear coherent diffusion model was proposed to reduce ultrasound speckle while preserving the appearance of structured regions and organ surfaces. The new technique has the advantages of robust parameter selection, speed of computation and maintenance of texture and organ surface. The new procedure has a large prospective in real-time ultrasound imaging enhancement and in assisting automated segmentation/calculation techniques.

J. Lu et. al. [4] "Contrast Enhancement of Medical Images Using Multi-scale Edge Representation" Knowledge suggest the existence of a link among the difference of a grayscale image and the gradient amount of intensity edges in the neighborhood anywhere the contrast is considered. This surveillance motivates the development of edge-based contrast development techniques. In this paper, we represent an effortless and successful method for image contrast improvement based on the multi-scale edge demonstration of images. The dissimilarity of an image can be improved simply by stretching or up scaling the multi-scale incline maxima of the image. This technique offers flexibility to selectively enhance features of unusual sizes and ability to control noise intensification. We present some experimental results from enhancing medical images and discuss the advantages of this wavelet approach over other edge-based techniques. Keywords: Contrast enhancement, multi-resolution processing, multi-scale edges, medical image processing, and wavelet transforms. Contrast Enhancement of Medical Images Using Multi-scale Edge Representation We have presented a description of an edge-based contrast enhancement method, and demonstrated its use with images drawn from three important medical imaging modalities. Edge-based enhancement certain striking advantages in excess of histogram-based technique. For example, we want contrast enhancement to take place along the direction perpendicular to edges in the image, and histogram-based techniques do not provide such spatial and orientation localization. The concept of edge-based contrast enhancement is not completely new; however, previously developed techniques all experience certain culties in implementing the basic idea. We have presented some evidence that many of these difficulties can be addressed in an effective way by using a wavelet based multiscale edge representation of images. Additionally, the wavelet approach era, among other advantages to be further explored, exibility to selectively enhance features of different sizes and in different locations, as well as the ability to control noise magnication

Yongjian $\mathrm{Yu}$ et. al. [5] "Speckle Reducing Anisotropic Diffusion" This paper present the origin of speckle reducing anisotropic diffusion (SRAD), a diffusion technique tailored to ultrasonic and microwave radar imaging applications. SRAD is the edge-sensitive diffusion for specked images, within the same approach that conventional anisotropic 
diffusion is the edge-sensitive diffusion for images corrupted with additive noise. We tend to 1 st show that the Lee and Frost filters may be cast as partial differential equations, and then we tend to derive SRAD by allowing edge-sensitive anisotropic diffusion inside this context. Even as the Lee and Frost filters exploit the coefficient of deviation in adaptive filtering, SRAD exploits the instant coefficient of variation, which is shown to be a perform of the local gradient magnitude and Laplacian operators. We tend to validate the new algorithmic rule using both synthetic and real linear scan ultrasonic imagery of the carotid artery. We tend to also demonstrate the algorithmic rule performance with real SAR data. The performance measures obtained by means that of simulation of carotid artery pictures are compared with 3 existing speckle reduction schemes. Within the presence of speckle noise, speckle reducing anisotropic diffusion excels over the standard speckle removal filters and over the conventional anisotropic diffusion technique in terms of mean preservation, variance reduction, and edge localization. We've got developed a nonlinear anisotropic diffusion technique, speckle reducing anisotropic diffusion, for removing multiplicative noise in imagery. not like other existing diffusion techniques that process log-compressed data, our technique processes the info $\}$ directly in order to preserve useful information in the image. We've shown that with a special window, both the Lee filter and also the Frost filter (which are usually used for filtering SAR imagery) will be solid into the framework of a diffusion technique. This observation has directed us to formulate a new adaptive edgepreserving PDE (in continuous image domain) tailored to specked imagery.

\section{METHOD}

To compare the performance of the projected noise reduction filter and other de-noising filters, a set of a popular speckle reduction technique was used and also the previously introduced quality metrics were extracted. Echocardiogram videos undergo some basic preprocessing before we start the method of feature point localization and coding. This includes extraction of the fan segment (which includes the actual image) with a rough alignment. For extraction of the region of interest, either manual or template matching based mostly automated technique will be used. Once the fan sector has been extracted, using the top, left and right extreme points, we tend to automatically align all the echocardiogram videos with each other via an affine remodel. The 3 points mentioned above are sufficient to calculate the transformation matrix. Although the classifier that we tend to intend to use, PMK primarily based SVM, is capable to handling small amount of image revolution incongruity, this preliminary alignment improves the discrimination ability of our system. These filters are SRAD, a TDLMS filter and some local statistical filters like the Lee Kuan , $3 \times 3$ median $\{$ and 3 and three $\} \times 3$ Wiener filters together with we'll use discrete wave transform.

In an echocardiogram video of a heart cycle, there are image regions (corresponding to completely different anatomical structures) which demonstrate significant amount of motion and other regions which don't. Furthermore, these regions are disparate for various viewpoints whereas similar for picture progression go to same viewpoints. To describe this info, we have a tendency to analyze the optical flow for echocardiogram video sequences computed using Demons algorithmic rule. The projected methodology consists of smoothing IVTCs assessed in every pixel. The IVTC signal may be explained by $\mathrm{p}(\mathrm{x}, \mathrm{y}, \mathrm{t})$ for the pixel in coordinate $(\mathrm{x}, \mathrm{y})$ at the edge of t. The expected procedure isn't limited to 1 cycle and may have any desired number of needed frames. Hence, the parameter $t$ is within the vary from 1 to $T$, within which $\mathrm{T}$ is the total number of frames which will be denoised. A sample IVTC signal from a set coordinate $(\mathrm{x}, \mathrm{y})$ of 128 consecutive frames. The signal variations are based on the intensity variations of each pixel within the time.

Three completely different points, which have different cardiac textures. These points are on the cardiac muscle, the ventricle border, and in the atrial cavity. The temporal sign from these unlike coordinates of $(\mathrm{x}, \mathrm{y})$ for $\mathrm{t}$ in the range of 1 to 250 of a real echocardiography sequence. As a result of the atrial cavity is darker than the cardiac muscle and ventricle border, the mean intensity of atrial cavity is smaller than the 2 different positions. As mentioned previously, when the backscattered acoustic pulses are received by the transducer they'll be in or out of phase. Hence, the granular patterns of speckle noise are the lighter and darker pixels on the image texture. These patterns correspond to high-frequency variations in the temporal signals. To know how the strength of a symbol is distributed in the frequency domain, spectral analysis of the temporal signals was accomplished. To observe the frequency elements, a single-sided amplitude spectrum was implemented. In a single-sided amplitude spectrum, the fast Fourier transform finds the frequency elements of a signal. Illustrates the equivalent Fourier transform of the original temporal signal in. evidently, by filtering the high-frequency elements of every temporal signal then replacing the smoothened signals in their positions, all pixels of all frames will be recreate in a similar way. To suppress high-frequency elements, completely different lowpass filters will be preferred. For ease, low difficulty, and proper correctness, we tend to choose a second-order Butterworth filter with a normalized cut off frequency of 0.25 . Note that there's no proscription against selecting different, more sophisticated low-pass filters. However, we should always have a trade-off between complexness and speed. The normalized cut-off frequency of 0.25 was chosen based on spectral info to decrease screaky elements and to stop blurring of fast-moving objects like the mitral valve. To demonstrates a filtered version of the signals. The amplitude spectrum of the filtered signals is illustrated.

\section{CONCLUSION}

We will introduced a novel scalable system for echocardiogram viewpoint classification which uses scale invariant features identify on edge filtered activity extent images and PMK based SVM and wavelet transform. The performance of this hybrid algorithm is better than the DWT based denoising. The performance of this hybrid approach can be improved using the redundant wavelet transforms such as undecimated wavelet transform, Dual tree complex wavelet transform and double density dual tree complex wavelet transforms for filtering the detail sub bands. 


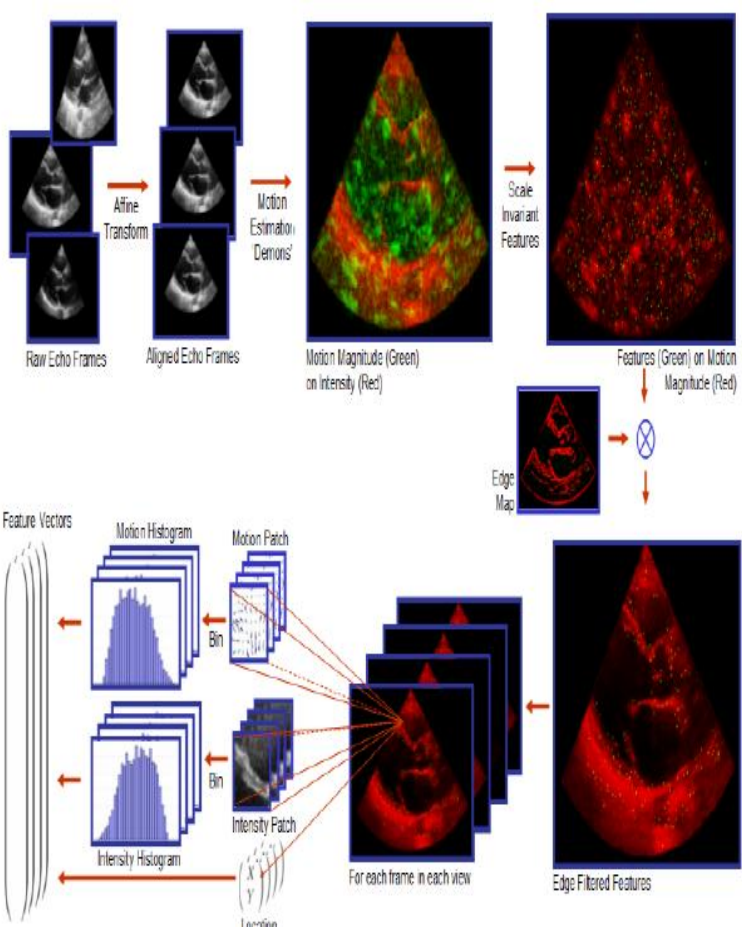

\section{REFERENCES}

[1] Parisa gifani, hamid behman, zahra alizadeh sani "Noise Reduction of Echocardiographic Images Based on Temporal Information" IEEE transaction on ultrasonics, ferroelectrics, and frequency control ,vol60

[2] C. B. Burckhardt, "Speckle in ultrasound b-mode scans," IEEE Trans. Sonics Ultrason., vol. SU-25, no. 1, pp. 1-6,
1978.

[3] K. Abd-Elmoniem, A.-B. Youssef, and Y. Kadah, "Realtime speckle reduction and coherence enhancement in ultrasound imaging via nonlinear anisotropic diffusion," IEEE Trans. Biomed. Eng., vol. 49, no. 9, pp. 997-1014, Sep. 2002.

[4] J. Lu and D. M. Healy Jr, "Contrast enhancement of medical images using multiscale edge representation," Proc. SPIE, vol. 2242, pp.711-719, 1996

[5] $\mathrm{Yu}$ and S. T. Acton, "Speckle reducing anisotropic diffusion,"IEEE Trans. Image Process., vol. 11, no. 11, pp. 1260-1270, Nov.2002.

[6] S. Finn, M. Glavin, and E. Jones, "Echocardiographic speckle reduction," IEEE Trans. Ultrason. Ferroelectr. Freq. Control, comparison vol. 58, no. 1, pp. 82-101, 2011

[7] D. Vatolin and A. Parshin, "Objective measurements of artifacts,produced by modern video coding standards," in Proc. Spring Conf. Computer Graphics, 2006.

[8] Z. Wang, A. C. Bovik, H. R. Sheikh, and E. P. Simoncelli, "Image quality assessment: From error visibility to structural similarity," IEEE Trans. Image Process., vol. 13, no. 4, pp. 600-612, Apr. 2004.

[9] X. Zong, A. Laine, and E. Geiser, "Speckle reduction and contrast enhancement of echocardiograms via multiscale nonlinear processing," IEEE Trans. Med. Imaging, vol. 17, no. 4, pp. 532-540, 1998.

[10] X. Hao, S. Gao, and X. Gao, "A novel multiscale nonlinear thresholding method for ultrasonic speckle suppressing," IEEE Trans. Med. Imaging, vol. 18, no. 9, pp. 787-794, 1999. 